\title{
Elliptic flow from collision geometry and rescattering
}

\author{
H. Bøggild* and Ole Hansen ${ }^{\dagger}$ \\ University of Copenhagen, The Niels Bohr Institute, Copenhagen, Denmark \\ T. J. Humanic ${ }^{\ddagger}$ \\ Department of Physics, The Ohio State University, Columbus, Ohio 43210, USA
}

(Received 20 December 2008; published 27 April 2009)

\begin{abstract}
Calculations of elliptic flow based on two initial state models of $\mathrm{Au}+\mathrm{Au}$ collisions at $\sqrt{s}=200 \mathrm{GeV} / \mathrm{n}$ coupled with a hadronic rescattering calculation are presented. The two initial state models used are a thermal model and a partonic model. Results from these calculations are compared with experiments and it is found that both initial state models give satisfactory representations of elliptic flow measurements, provided that the rescattering is started early enough in the collision process. It is also found that the present hadronic model studies do not show the jet suppression observed experimentally.
\end{abstract}

DOI: 10.1103/PhysRevC.79.044912

PACS number(s): 25.75.Ld, 25.75.Dw, 25.75.Gz

\section{INTRODUCTION}

In high-energy heavy-ion collisions flow phenomena have been used to explain the observation of the shapes of transverse momentum spectra and of azimuthal asymmetries in the momentum distributions [1,2]. In a previous article [3] it was shown that rescattering would change the slopes of transverse momentum spectra such that the slope became flatter with increasing mass of the particle species in good accordance with experimental data and irrespective of whether the input model data to the rescattering code [4] came from a thermal-like event generator or from a partonic based generator. The main aim of the present report is to investigate if the same is true for azimuthal asymmetries in the particle distributions, measured by the strength of the second harmonic of the distributions relative to the reaction plane, the so-called elliptic flow or $v 2$ value, observed systematically in $\mathrm{Au}+\mathrm{Au}$ collisions at 130 and $200 \mathrm{GeV} /$ nucleon experiments (see Refs. [5] and [6]). Many theoretical studies of elliptic flow have been published based on a variety of models ranging from fluid dynamics (see, e.g., Ref. [7] and references therein), covariant transport theory (e.g., Ref. [8]), the UrQMD approach [9,10], the PHSD approach (parton-hadron-strings-dynamics) [11], and the AMPT (a multiphase transport) model [12-14]. These approaches are complete, starting on the parton level and going through a dynamical development to the final hadronic phase, whereas the present approach is much simpler, and also less realistic, only encompassing a hadronic description from the first collision moment to freeze-out. The goal here is to learn to which extent such a simplistic description can reproduce experimental data on elliptic flow and under what parameter conditions this may be attained.

It will be shown that hadronic rescattering can produce observed magnitudes of $v 2$ values as well as their dependencies on the centrality of the collisions, the particle

\footnotetext{
*boggild@nbi.dk

†ohansen@nbi.dk

†humanic@mps.ohio-state.edu
}

species, and the distributions in pseudorapidity and transverse momentum.

\section{EVENT GENERATORS AND RESCATTERING}

\section{A. The thermal-like generator}

The thermal-like generator for $\mathrm{Au}+\mathrm{Au}$ collisions has not been changed from the description given previously [3] and only a very brief outline of the essentials is given here. Four-momentum vectors are created from source distributions in rapidity, one vector (particle) for each source, following a standard Boltzmann probability prescription in energy and with spherically uniform direction choices in the source rest frame. The source distributions consist of three Gaussian distributions, one centered at midrapidity, one at forward rapidity (see Table I), and the last at the same backward rapidity. The geometry of the three source distributions together with the relative number of particles from the source centers (forward and backward equal) were fitted to rapidity density data from experiment. A Au + Au event is constructed by generating 5301 pions, 1000 kaons, 530 protons, and 269 antiprotons in all 7100 particles at a common temperature parameter of $270 \mathrm{MeV}$. Of these only particles with rapidity $-6.5<y<6.5$ are fed into the rescattering program, a roughly $4 \%$ reduction, undertaken to approximate energy and momentum conservation corresponding to $200 \mathrm{Gev} /$ nucleon collisions. A thermal-like model does not by itself maintain the normal conservation laws. The events in different model-runs are not identical because the random number generator [15] is started at a different point for each event. The generator has an energy cutoff in the Boltzmann distributions near 15 times the temperature, i.e., at $4 \mathrm{GeV}$, corresponding to a maximum transverse momentum of $3.89 \mathrm{GeV}$ for protons and just below $4.0 \mathrm{GeV}$ for pions. This cutoff was of no consequence in Ref. [3] but renders the thermal-like generator impractical for the high $p_{t}$ physics discussed later. The probability for creating a particle with transverse momentum at e.g. $5 \mathrm{GeV}$ in the Boltzmann distribution is on the order of $10^{-8}$ and such 
TABLE I. Parameters of the thermal model. $y$-mid is the rapidity of the middle Gaussian source center, $y$-forward is that of the forward center, and the backward center has the corresponding negative value. The $\sigma$ 's are the Gaussian widths of the source distributions. $N_{+} / N_{0}$ is the ratio of particles in the forward source distribution and the number in the middle distribution and $\sigma$-forward $=\sigma$-backward. The ratio in the backward distribution is $N_{-} / N_{0}=N_{+} / N_{0}$.

\begin{tabular}{lcc}
\hline \hline Particle & Quantity & Values \\
\hline All & $y$-mid & 0.00 \\
All & $y$-forward & 3.50 \\
All & $\sigma$-mid & 1.50 \\
All & $\sigma$-forward & 2.00 \\
$p$ & $N_{+} / N_{0}$ & 0.95 \\
$\bar{p}$ & & 0.18 \\
$\pi$ & & 0.40 \\
$\mathrm{~K}$ & & 0.45 \\
\hline \hline
\end{tabular}

particles would not contribute in a significant way even if the cutoff was changed.

\section{B. The partonic generator}

In the partonic generator a simplification in the modeling of the $A+A$ collisions was made. Instead of colliding bunches (trains) of nucleons as described in our previous work [3] we now in a more straightforward way simulate the collisions of two spheres, each with $A$ nucleons at random positions and with radii of $R=1.12 A^{1 / 3}$. As the nuclei pass through each other the nucleons interact with known inelastic cross sections, lose energy, interact again (as wounded nucleons), etc., until they escape as wounded nuclei decaying to pions and nucleons. This change in procedure has little influence on our previously published results. The individual nucleon-nucleon collisions are handled as in our previous work, i.e., each resulting in four excited clusters, two forward baryonic systems (the wounded nucleons) and two central clusters. The energies of the forward systems are obtained from a simple longitudinal phase space distribution and in the end each escaping forward wounded nucleon decays to a nucleon and a forward excited mesonic cluster. The central excited clusters scatter with a QCD inspired angular distribution and decay as if they were originating from $e+e-$ collisions. For details see Ref. [3].

\section{The hadronic rescattering calculation}

The rescattering code used here is in most ways the same as that used in Ref. [3] and in earlier publications (see, e.g., Ref. [4]), although some changes have been made. First, the code was upgraded to use double precision throughout and the random number generator was changed to one with a longer cycle and fewer correlation problems than the standard Fortran routine; it is documented in Ref. [16]. This change did not have a significant effect on the results, showing the good numerical stability of the code. An error in the treatment of inelastic scattering was corrected, which mostly affected particles with $p_{t}>3 \mathrm{GeV}$ (which was not important for previous work that focused on lower $p_{t}$ studies). Two further changes were introduced, both dealing with the initialization of the rescattering. The first was a change in the geometry for finite impact parameters (denoted $b$ ) and has no influence on central collisions as reported in Ref. [3]. The change includes all particles in the collision that is swept by under the movement of one sphere through the other, containing the initial particles, while previously [4] only particles contained in the overlap of both spheres in their middle position $(z=0$ along the collision axis) were included. This results in more particles participating in the rescattering process as compared with previous finite impact parameter applications. The second change was of somewhat more substance. In earlier use of the rescattering code the beginning coordinates for the rescattering were determined as

$$
z=\tau_{0}\left(p_{z} / m\right), \quad t=\tau_{0}(e / m)
$$

and $x$ and $y$ were chosen randomly within the overlap region. $(x, y, z)$ are the space coordinates of the particle with the $z$ axis in the collision direction and one nucleus with the center at $(-b / 2,0,0), p$ is the momentum, and $e$ is the energy. The other nuclear center is moving parallel to the $z$ axis at a distance $b$ from the other center in the $x$ direction and with $y=0$. Thus the particle has moved in the $z$ (boost) direction but not in the transverse direction before rescattering is started a time $t . \tau_{0}$ was $1.0 \mathrm{fm} / \mathrm{c}$ in Ref. [3], roughly a tenth of the value expected by the Bjorken [17] model, after which the above beginning conditions were chosen. In the present report the particles have been allowed to move in the transverse plane also during the time $t$, so Eq. (1) has been substituted with

$$
\begin{aligned}
& x=x_{0}+\tau_{0}\left(p_{x} / m\right), y=y_{0}+\tau_{0}\left(p_{y} / m\right), \\
& z=\tau_{0}\left(p_{z} / m\right), t=\tau_{0}(e / m),
\end{aligned}
$$

where $\left(x_{0}, y_{0}\right)$ were chosen randomly within the overlap region.

The particle is hence further toward the border of the collision zone when rescattering is allowed to start than in the previous procedure and the idea of an initial flow in the boost direction, as advocated in Ref. [17], has been abandoned. This change gives rise to the use of smaller values of $\tau_{0}$ to reproduce previous results with the rescattering model.

\section{SYSTEMATICS OF MODEL v2 VALUES}

The $v 2$ value is, following Ref. [18], defined by

$$
v 2=\left\langle\cos 2\left(\phi_{i}-\Psi\right)\right\rangle,
$$

where $\phi_{i}$ is the azimuthal angle of the momentum vector of particle $i$ and the $x-z$ plane and $\Psi$ is the azimuthal angle of the reaction plane with the $x-z$ plane and the averaging is over all particles $i$ in an event. The reaction plane in all model $v 2$ values calculated from the thermal-like generator is the $x-z$ plane, i.e., $\Psi=0$. For the partonic generator both this method and one where the reaction plane is determined for each event from the model data by the prescription in Ref. [18], i.e., Eq. (4) 




FIG. 1. $v 2$ versus $p_{t}$ for different values of $\tau_{0}$ with input from the thermal generator. The figure is for pions and represents 5000 events.

are used, where

$$
\Psi=\left(\arctan \frac{\Sigma w_{i} \sin \left(2 \phi_{i}\right)}{\Sigma w_{i} \cos \left(2 \phi_{i}\right)}\right) / 2,
$$

where $w_{i}$ are weights, here taken as $p_{t}$ of the individual particle. For the analysis of particle $i$ its contribution to the sums in Eq. (4) was subtracted, following Ref. [18]. Finally the $v 2$ values given are averaged over the event sample used in the model calculations.

\section{A. Dependence on $\boldsymbol{\tau}_{\mathbf{0}}$, mass, and impact parameters}

The time parameter $\tau_{0}$ of Eq. (2) determines when rescattering starts in space and time, and therefore the amount of rescattering that takes place while the particle density is high. Figures 1 and 2 demonstrate the dependence on $\tau_{0}$ for both event generators at an impact parameter of $b=8.0 \mathrm{fm}$. For the thermal-like generator the highest values of $v 2$ occur for the lowest $\tau_{0}$ and vice versa. For high values of $\tau_{0}$ the $v 2$ values fall off with increasing $p_{t}$ after about $1 \mathrm{GeV}$, while for the lowest $\tau_{0}$ value $v 2$ stays flat with $p_{t}$ at the maximum value, a general trend for the thermal-like generator valid for other finite values of impact parameter $b$. For the partonic generator the systematics are somewhat different, in that the $v 2$ always decreases with $p_{t}$ for $p_{t}$ larger than about $1.8 \mathrm{GeV}$, though to a lesser extent for the lower $\tau_{0}$ values. Also $\tau_{0}=0.1 \mathrm{fm}$ does not lead to a $v 2$ value higher than that obtained with $\tau_{0}=$ $0.2 \mathrm{fm}$. This again holds true for other impact parameters. Finally it may be noted that the low $p_{t}$ behavior is different for each $\tau_{0}$ in the partonic generator case while for the thermal-like generator the slopes are quite similar at small $p_{t}$.

The rescattering mechanism creates the azimuthal asymmetry, which is demonstrated in Fig. 3, where the black squares show $v 2$ values produced by the thermal-like generator with the geometry selection of the rescattering code but with

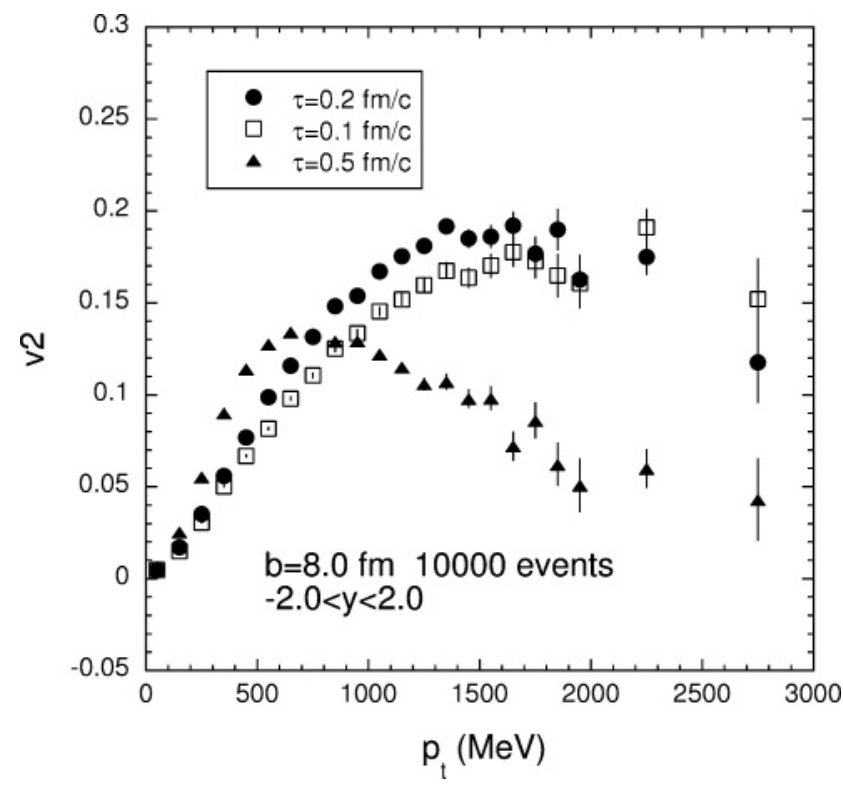

FIG. 2. $v 2$ versus $p_{t}$ for $b=8.0 \mathrm{fm}$ and $\pi$ mesons with different values of $\tau_{0}$. Input to the rescattering code is from the partonic generator with 10000 events.

the rescattering itself turned off; all points are near $v 2=0$, while the points with rescattering have nonzero $v 2$ values. The impact parameter used is $b=8.0 \mathrm{fm}$, corresponding to a rather peripheral collision. The second feature that can be seen from Fig. 3 is the mass dependence of $v 2$. The largest values obtained are for nucleons ( $\mathrm{N}$ in the figure), lower values are for $\mathrm{K}$ mesons, and the smallest values are for $\pi$. The nucleon values increase with increasing $p_{t}$ while for $\pi$ the values tend to fall off at the highest $p_{t}$. It is also clear that the nucleon values increase more slowly toward the maximum than those

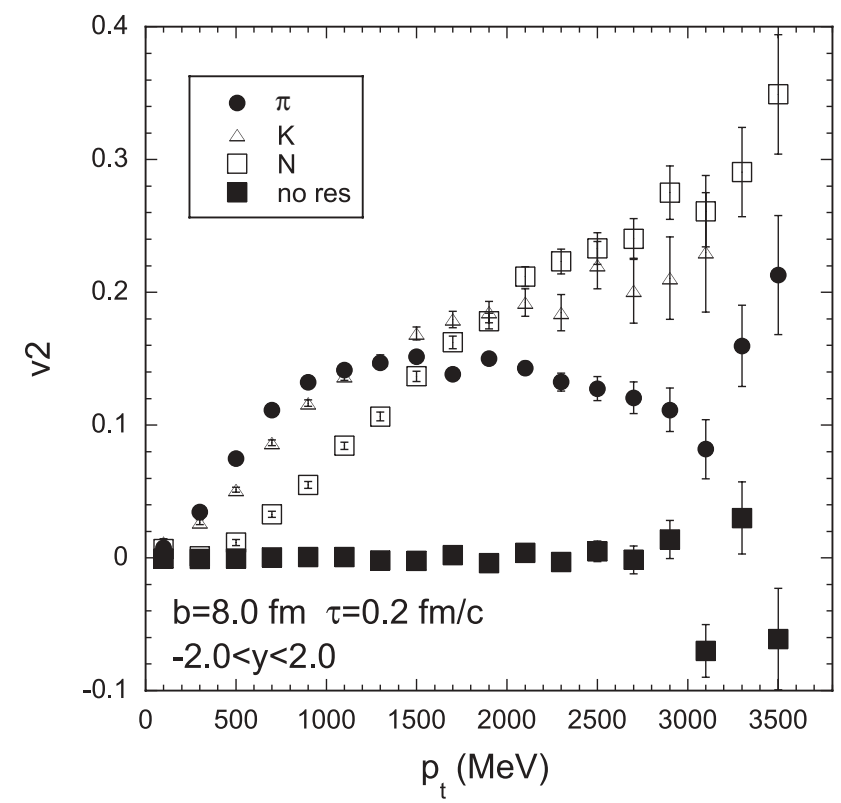

FIG. 3. $v 2$ versus $p_{t}$ for $b=8.0 \mathrm{fm}$ and different particle species, as well as for all particles with rescattering turned off. Input to the rescattering code is from the thermal-like generator with 5000 events. 


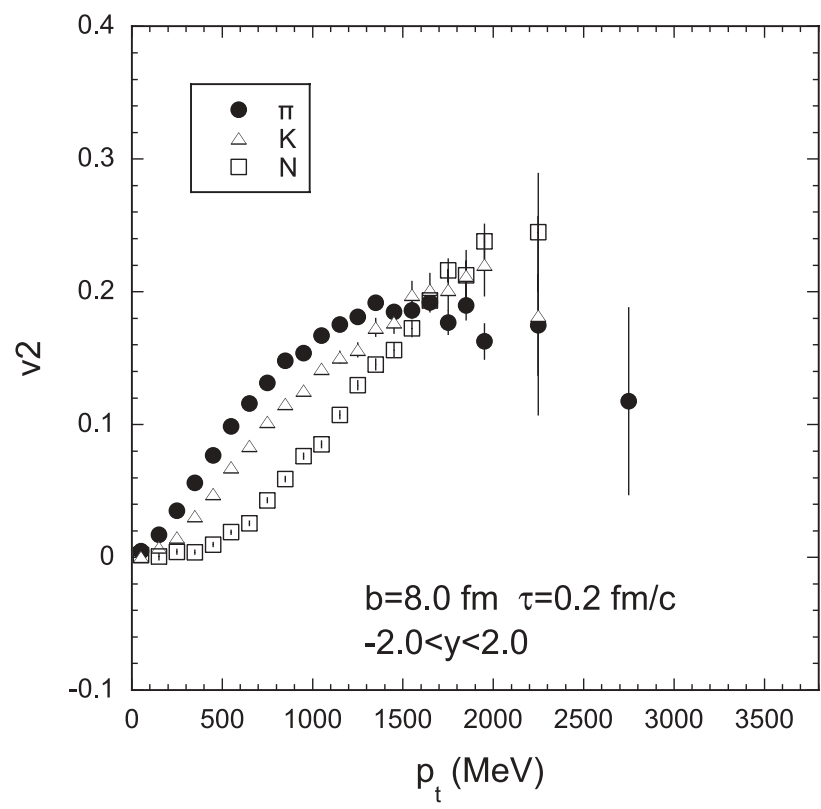

FIG. 4. $v 2$ versus $p_{t}$ for $b=8.0 \mathrm{fm}$ and different particle species. Input to the rescattering code is from the partonic generator with 10000 events.

for the lighter particles. These trends are similar at other values of $\tau_{0}$ and impact parameter $b$ also. Figure 4 shows the mass systematics for the partonic generator. As for the thermal-like case the $v 2$ values for pions increase faster with $p_{t}$ than those for kaons and nucleons, but all three particles reach roughly the same $v 2$ value at about $1.7 \mathrm{GeV}$. The $v 2$ flattens out for the pions but continues rising for the kaons and nucleons, as for the thermal model.

Figure 5 shows the $p_{t}$ dependence of $v 2$ from the thermal-like generator for three different impact parameters, $b=0.0, b=4.0$, and $b=8.0 \mathrm{fm}$, analyzed without particle identification, i.e., rather close to the $\pi$ behavior at $p_{t}$ up to about $2.5 \mathrm{GeV}$ and more as nucleons and $\mathrm{K}$ mesons at higher $p_{t}$ (see also Fig. 3). The $b=0.0 v 2$ are zero or very close, while they are higher for $b=4.0$ and maximum for $b=$ $8.0 \mathrm{fm}$. Even if the rescattering creates the azimuthal asymmetries they vanish for the central collisions. The impact parameter systematics for the partonic generator (not shown) are similar, increasing maximum $v 2$ value for increasing impact parameter.

The overall trends shown here are quite similar to what has been found for other models, Refs. [7-14].

\section{B. Reaction plane and event plane}

The $p_{t}$ dependence of $v 2$ appears to be somewhat different for the two event generators. $v 2$ was analyzed with the partonic generator both with the $x-z$ plane as the reaction plane and with a reaction plane determined event-by-event using Eq. (4) as demonstrated in Fig. 6. It can be concluded from the figure that the difference between the two definitions of the reaction plane causes very small differences in $v 2$ and is therefore hardly the cause of the different $p_{t}$ behavior between the two event generators.



FIG. 5. $v 2$ versus $p_{t}$ for three different values of the impact parameter $b$. No particle identification was used in the $v 2$ analysis and the input was from the thermal-like generator. Five thousand events were used for $b=8.0 \mathrm{fm}, 2500$ for $b=4.0 \mathrm{fm}$, and 600 for $b=$ $0.0 \mathrm{fm}$.

\section{Dependence on pseudorapidity}

The two generators have, so to speak, perfect particle identification, and the $v 2$ dependence on rapidity for each particle species can be calculated. Pseudorapidity has been used here rather than rapidity, for the reason that much of the experimental data published so far use that variable. The

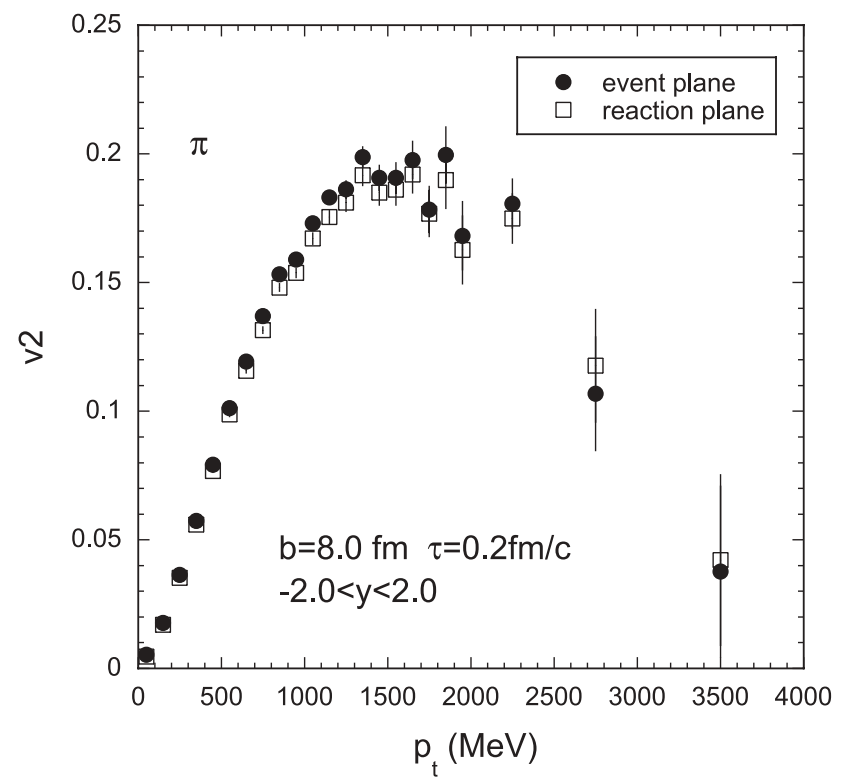

FIG. 6. $v 2$ versus $p_{t}$ for the two different definitions of the reaction plane, either the $x-z$ plane or the event-by-event definition following Eq. (4). The input to the rescattering code was from the partonic generator with 10000 events. 
general shapes of the $v 2$ distributions with pseudorapidity,

$$
\eta=\ln \left(\left(p+p_{z}\right) / p_{t}\right),
$$

are similar for both generators as can be seen from Figs. 8 and 9 below, where data and model predictions are compared. The shapes neither depend strongly on impact parameter nor depend on whether an interval of impact parameters is used rather than a single one. The maximum value of $v 2$ is reached for $\eta=0$ and $v 2$ decreases to both sides and with a rather pronounced shoulder structure between $\eta=2$ and $\eta=3.5$. The maximum value depends on impact parameter selection as can also be seen from the figures.

\section{COMPARISON WITH EXPERIMENT}

The STAR, PHOBOS, and PHENIX experiments at RHIC have published $v 2$ data (see, e.g., Refs. [5,6,19], and [20]) that are in good agreement with one another. We have chosen to use the data from the first three references for the comparisons below.

\section{A. Simulation of experimental centrality cuts}

Both the PHOBOS and the STAR experiments use measured charged particle multiplicities for centrality cuts. The simulations used here employ overall multiplicities after rescattering, because charge does not enter any of the generators. A sample of 10000 events was made with the partonic generator with impact parameters from 0 to $b_{\max } \approx 13 \mathrm{fm}$, corresponding to twice the radius of a $\mathrm{Au}$ nucleus, with a probability distribution $p(b)$

$$
d(p(b)) / d b=\left(2.0 / b_{\max }^{2}\right) b .
$$

For the thermal-like generator a similar sample of 10000 events was made also using Eq. (6) for the impact parameter selection, and because the algorithm used an unreasonably long time to handle events with very small multiplicities (of a few), the $b$ selection was stopped at $12.50 \mathrm{fm}$. For both generators the distribution of the corresponding multiplicities was turned into a multiplicity probability distribution and integrated in steps of 100 from 0 until an area of 1.000 was reached, just below a multiplicity of 8000 . The experimental cuts in both PHOBOS and STAR are given as percentages of the total inelastic cross section and in our case the multiplicities corresponding to those percentages were used for the event selection. For the thermal-like generator a procedure with direct impact parameter selection from the minimum bias sample corresponding to the same cross section percentages was also performed with results in very close agreement with those of the multiplicity method.

\section{B. Comparison to results from PHOBOS}

The comparison to PHOBOS results is based on Ref. [6]; the cross section cuts used in the centrality selection can be found in Table II. The translation to the recattering multiplicities used here are also given in Table II. The first comparison is given in Fig. 7 showing the PHOBOS $v 2$ data versus $p_{t}$ from their track based method of analysis of particles in the
TABLE II. Multiplicites used in the simulation of PHOBOS centrality cuts. The left-most column states the name used in Ref. [6] for the centrality cut and the next column gives the pecentage of the total inelastic cross section corresponding to the cut (see Ref. [6]). The next four columns are the overall multiplicity cuts used after rescattering in the comparisons, charged and uncharged particles in

\begin{tabular}{|c|c|c|c|c|c|}
\hline \multirow[t]{2}{*}{ Centrality } & \multirow[t]{2}{*}{$\% \sigma_{\text {inel }}$} & \multicolumn{2}{|c|}{ Thermal generator } & \multicolumn{2}{|c|}{ Partonic generator } \\
\hline & & Min. & Max. & Min. & Max. \\
\hline Min. bias & 100 & 0 & 8000 & 0 & \\
\hline Central & $3-15$ & 4300 & 6300 & & \\
\hline Mid-central & $15-25$ & 3100 & 4300 & 3200 & \\
\hline Peripheral & $25-50$ & 1300 & 3100 & 1350 & 3200 \\
\hline $\begin{array}{l}\text { Most } \\
\text { central 50\% }\end{array}$ & $0-50$ & 1300 & 7450 & & \\
\hline
\end{tabular}
$4 \pi$ geometry.

interval $0<\eta<1.5$ and the corresponding results from the two generators.

The agreement between data and models is quite good, although the thermal model underpredicts the data near $2 \mathrm{GeV} / \mathrm{c}$. No particle identification has been used in the experiment or in the models.

The comparisons for the pseudorapidity dependence are shown in Figs. 8 and 9. Figure 8 shows the PHOBOS data for the peripheral centrality cut and the "hit based analysis" of Ref. [6] and the $v 2$ values from the partonic generator. The overall shapes of the two distributions agree reasonably well but the absolute $v 2$ values are systematically underpredicted by about 0.01 . The shoulder structure of the generator results is not confirmed by the experimental data, but data are lacking to an extent in the most sensitive regions of $\eta$. The results

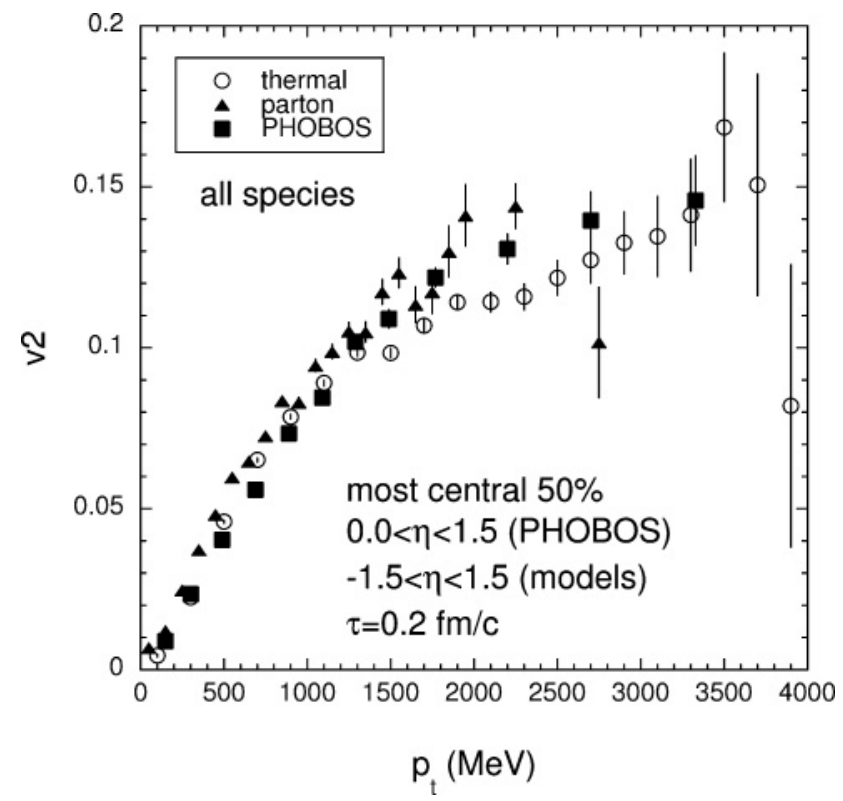

FIG. 7. $v 2$ comparison between PHOBOS and the two generators for the most central $50 \%$ centrality cut of Table II. See the text for further details. 




FIG. 8. $v 2$ comparison between PHOBOS and the partonic generator for the peripheral centrality cut in Table II. See the text for further details.

for the thermal-like generator for the peripheral cut (not shown) is close in absolute $v 2$ values as compared with the PHOBOS results of Fig. 8. The comparison for the central cut is shown in Fig. 9, this time with generator results from the thermal-like generator, and here the absolute values are fairly well predicted, while the partonic generator (not shown) somewhat underpredicts the absolute $v 2$ magnitude. Again the shoulder structure in the model data is not confirmed by experiment. The absolute $v 2$ values from the two generators do not agree with one another as has also been seen above.



FIG. 9. $v 2$ comparison between PHOBOS and the thermal-like generator for the central cut of Table II. One thousand two hundred and eighty events fell in the multiplicity range of the central trigger. See the text for further details.
TABLE III. Multiplicities used in the simulation of STAR centrality cuts. The left-most column gives the centrality percentages as used in Ref. [5] and the following columns state the overall multiplicity limits used after rescattering to mimic the experimental cuts.

\begin{tabular}{lrrrrr}
\hline \hline $\begin{array}{l}\text { Centrality } \\
\% \sigma_{\text {inel }}\end{array}$ & \multicolumn{2}{c}{ Thermal generator } & & \multicolumn{2}{c}{ Partonic generator } \\
\cline { 2 - 3 } & Min. & Max. & & Min. & Max. \\
\hline $5-10$ & 5100 & 5900 & & & \\
$20-30$ & 2700 & 3750 & & & \\
$60-70$ & 500 & 800 & & 15 & 8500 \\
$\approx 100$ & 15 & 8000 & & 15 \\
\hline \hline
\end{tabular}

The agreement with the PHOBOS data can be improved by appropriate changes in the limiting multiplicity cuts, for the partonic generator. However, quantitative agreement with the data is not an important goal considering the rather simplistic nature of the generators and the trigger simulations.

\section{Comparison to results from STAR}

The STAR experiment presents results on $v 2$ measurements for identified particle species and therefore provides an opportunity for a more detailed test of the ability of the rescattering procedure to reproduce experimental results. Limits in the particle identification possibilities in STAR also influence the scope of this comparison.

The STAR experiment uses different centrality and pseudorapidity cuts from the PHOBOS experiment. The simulation of the STAR trigger cuts uses the same procedure as was used for the PHOBOS case as explained above. The multiplicity cuts used in the simulation are shown in Table III based on the definitions in Ref. [5], as quoted in the article by J. Adams et al. [5], Table II. In addition the pseudorapidity satisfied $-1.3<\eta<1.3$ (Table I of Adams et al. [5]) and the minimum bias rescattering events had multiplicities larger than 15 corresponding roughly to the STAR cut in charged multiplicity of 10 (their Table I). This simulation of the STAR cuts is not expected to be an accurate mimicking of the experimental procedures but only a reasonable approximation.

A comparison between STAR results for $\Lambda+\bar{\Lambda}$ versus $p_{t}$ and rescattering results for nucleons and antinucleons, both from the thermal-like generator and for the partonic generator, is shown in Fig. 10. The centrality cut, labeled "minimum bias" in Adams et al. [5], corresponds to the centrality $\approx 100 \%$ in Table III. The figure covers $p_{t}$ up to $6.0 \mathrm{GeV}$ for the experiment and to about $3.5 \mathrm{GeV}$ for the generator results. Nucleon data exist from the PHENIX experiment [20] up to $\approx 3.3 \mathrm{GeV}$ and they agree with the $\Lambda+\bar{\Lambda}$ results of Fig. 10, (see, e.g., Fig. 10 of Adams et al. [5]). Both generator results agree with the $\Lambda$ data quite well in the entire $p_{t}$ range covered.

A comparison of $v 2$ results for $\pi$ mesons and nucleons for low $p_{t}$ are shown in Figs. 11 and 12, respectively, where STAR data (Figs. 7(a) and 7(c) of Ref. [5]) are compared to results from the thermal-like generator. Three different centrality bins were used for the $\pi$ mesons, a central cut (5-10\%), 


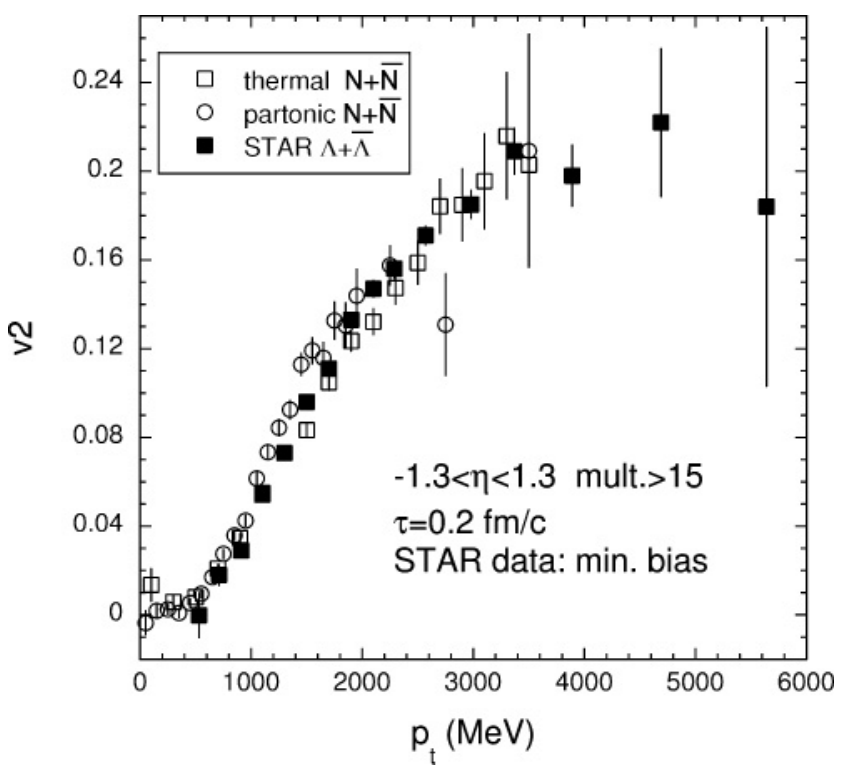

FIG. 10. $v 2$ comparison between $\Lambda+\bar{\Lambda}$ data from STAR [5] and rescattering results from both generators for $N+\bar{N}$ as a function of $p_{t}$. Centrality is $100 \%$, a minimum bias trigger (Table III). Ten thousand events were used for both generators. See the text for further details.

a peripheral cut $(60-70 \%)$, and a middle choice (20-30\%) as detailed in Table III. The agreement between experiment and the rescattering procedure is good. Figure 12 shows the comparison between $\bar{p}$ results from STAR and $N+\bar{N}$ from the thermal-like generator and only for the central and peripheral cuts. Even though both experiment and generator results suffer from rather poor statistics a reasonable agreement between the two sets of data is evident. The middle cut is not shown because

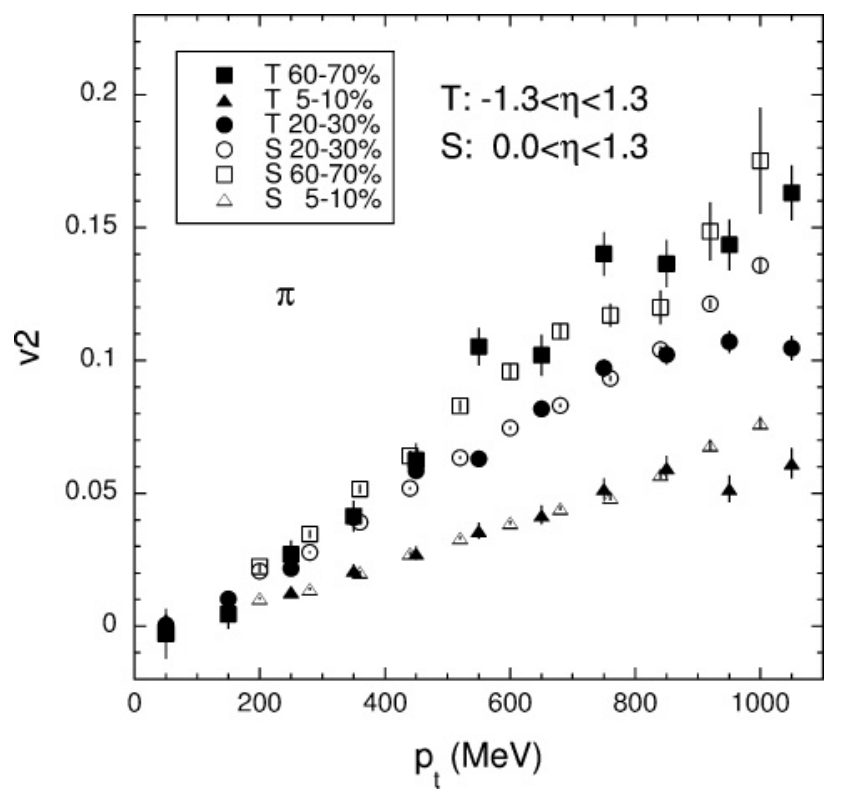

FIG. 11. $v 2$ comparison between $\pi$-meson data from STAR [5] (denoted $\mathrm{S}$ in the figure) and rescattering results from the thermal-like generator $(\mathrm{T})$ as a function of $p_{t}$ for three different centrality cuts. See the text for further details.

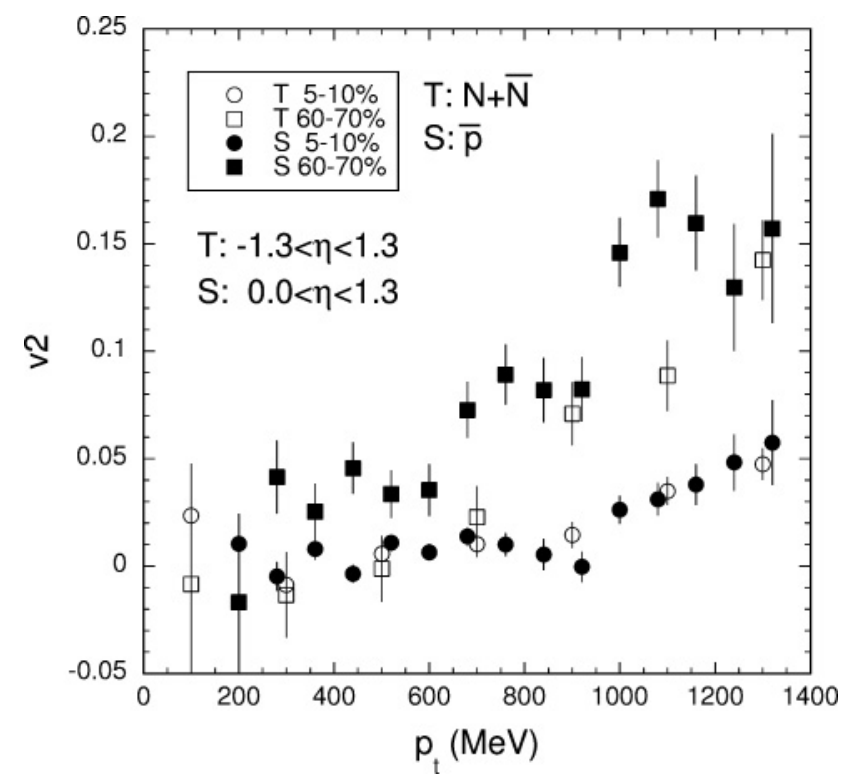

FIG. 12. $v 2$ comparison between $\bar{p}$ data from STAR [5] (denoted $\mathrm{S}$ in the figure) and rescattering results for $N+\bar{N}$ from the thermallike generator (denoted $\mathrm{T}$ ) as a function of $p_{t}$ for two different centrality cuts. See the text for further details.

the results run together with those of the neighbor cuts due to the limited statistics.

The data from the three RHIC experiments, STAR, PHOBOS, and PHENIX, are plentiful and would allow many more comparisons. The contents of Figs. 7-12, however, demonstrate the main trends to be learned from the schematic models used here. At $p_{t}$ below $\approx 1 \mathrm{GeV}$ the agreement between the thermal results and the STAR data is good for different centrality cuts and for different particle species. The agreement holds for the minimum bias cut up to $\approx 3.5 \mathrm{GeV}$ for $N+\bar{N}$ as shown in Fig. 10 for both generators, and it holds reasonably well for unidentified particles up to $\approx 3.5 \mathrm{GeV}$ and the most central 50\% (Fig. 7). The pseudorapidity distributions from both generators show a pronounced shoulder structure near $\eta= \pm 3$ that is not evident in the PHOBOS data (Figs. 8 and 9), while otherwise the agreement between data and rescattering results is reasonable.

\section{DISCUSSIONS}

\section{A. Physical meaning of $\tau_{0}$}

The time frame used in the original collision picture by Bjorken [17] has hadronization taking place after about $10 \mathrm{fm} / \mathrm{c}$ of longitudinal fluid expansion. In the hydrodynamical picture, as e.g., used by Heinz in Ref. [7], the hadronization starts earlier, around $4 \mathrm{fm} / \mathrm{c}$, where the mixed phase has developed from pure quark-gluon plasma. The azimuthal asymmetry, however, starts already in the quark-gluon phase and is carried over into the hadron phase, where the momentum space asymmetry is fully developed after about $7 \mathrm{fm} / \mathrm{c}$. The times in the presently used rescattering description are different. First, there is no quark-gluon plasma phase-the particles are hadrons from the beginning. In the model of Eq. (2) 




FIG. 13. $v 2$ dependence on time $t$ in $\mathrm{fm} / \mathrm{c}$, where the initial time for the onset of a possible rescattering event for the particle is defined in Eq. (2). The impact parameter is $8.0 \mathrm{fm}, \tau_{0}=0.2 \mathrm{fm} / \mathrm{c}$, and events from the partonic generator were used. The figure is discussed in the text.

the particles move with the event generator momenta until rescattering sets in at time $t$. The azimuthal asymmetry is created by the rescattering, quite as in the hydrodynamical picture. Figure 13 illustrates the development of $v 2$ in time for the partonic generator and all particle species. The $v 2$ develops from zero rather steeply and flattens out near $10 \mathrm{fm} / \mathrm{c}$ to reach a plateau from about $20 \mathrm{fm} / \mathrm{c}$. Very closely the same time evolution is found in Ref. [4] for a different thermal-like model and the Eq. (1) start conditions with $\tau_{0}=1.0 \mathrm{fm} / \mathrm{c}$, as in Ref. [3]. This time development is slower than the time development of the transverse momentum asymmetry estimated in Ref. [7] (see Fig. 14 of the reference). The PHSD model [11] and the AMPT model [14] show similar fast time developments, though in the AMPT case shown only for partons. The time development in the present hadronic picture is started by the choice of $\tau_{0}$, the parameter that determines the onset of rescattering and as demonstrated earlier, values larger than $0.2 \mathrm{fm} / \mathrm{c}$ leads to smaller $v 2$ values, a trend that is also found in the UrQMD model (Fig. 9 of Ref. [9]). Early onset of scattering is required in the hadronic picture to reach the magnitude of the experimentally observed $v 2$ values, and the same holds true for all the other models cited, in which scattering is introduced at the partonic level.

The $v 2$ development in the present picture starts early in the collision process and reasonable agreement with experiment can be obtained without any phase transition and without any fluidity concepts in the modeling.

\section{B. Origin of $\boldsymbol{v} 2$ in the rescattering}

It was demonstrated above that no momentum azimuthal asymmetry exists without rescattering, a feature that arises by

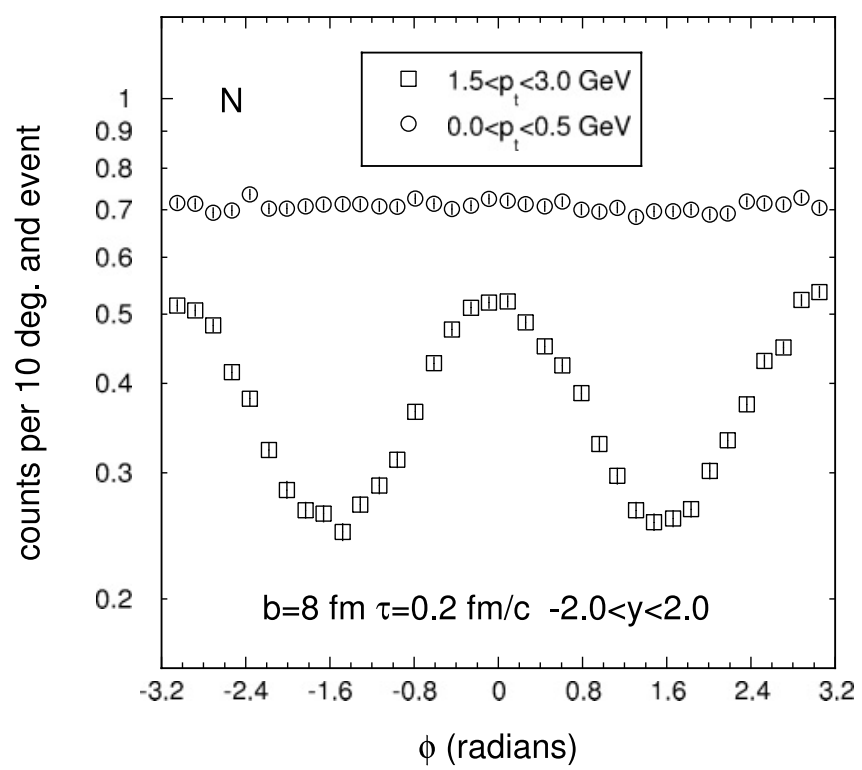

FIG. 14. Angular distribution of particles in azimuthal angle $\phi$ from the thermal model with 5000 events for two different $p_{t}$ intervals.

construction. In both event generators the momentum vectors are created with an even distribution of the azimuthal angle $\phi$ with respect to the $x-y$ plane, a symmetry that is not broken by boosts in the $z$ direction and is also maintained by the rescattering code after geometry selection as long as the rescattering processes themselves are turned off. It has also been shown that central collisions with rescattering do not produce an azimuthal momentum asymmetry, so both a geometry cut from a finite impact parameter and rescattering are needed to produce a $v 2$.

The initial distribution of particles before any rescattering is symmetric in both $x$ and $y$ directions (the azimuthal directions) by construction, with maximum density at $x=0$ and $y=0$ for all impact parameters. A study with the thermal generator and an asymmetric initial $x$ distribution with a maximum near $x=1.6 \mathrm{fm}$ gave the same systematics as described above and about $10 \%$ larger $v 2$ values. In the calculations shown here the initial value of $z$ before rescattering (at time zero) is $z_{0}=0$. Calculations were made with the partonic generator where the initial $z$ value was smeared over $2 \mathrm{fm}$, and no significant changes were issued. It may be concluded that the results presented here are robust toward small changes in the initial particle distribution within the almond shaped geometry from the collision process.

The full width at half maximum (FWHM) of the initial $x$ distribution at time zero is narrower than the FWHM in the corresponding $y$ distribution, e.g., $4.0 \mathrm{fm}$ versus $6.4 \mathrm{fm}$ for $b=8.0 \mathrm{fm}$, and hence the probability for rescattering of a particle moving in the $x$ direction is smaller than for one moving in the $y$ direction. Rescattering moves more particles out of the $y$ direction than out of the $x$ direction, resulting in an azimuthal asymmetry in the momentum distribution, demonstrated by Fig. 14. The figure shows the azimuthal final particle distribution after rescattering at low $p_{t}$ (top, a flat distribution) and at high $p_{t}$ (middle, a cosine-like distribution) for nucleons and $b=8.0 \mathrm{fm}$. At high $p_{t}$ more particles 




FIG. 15. Ratios of invariant cross sections for different impact parameters $b$ versus $p_{t}$ from the partonic generator. The ratios are the cross sections with rescattering divided by the corresponding cross sections without.

emerge at $\phi=0, \pi$, and $-\pi$ than at $\phi=\pi / 2$ and $-\pi / 2$. The transverse asymmetry arises in the present model from the collision geometry combined with the kinematics in the rescattering.

\section{High $p_{t}$ behavior}

While the hadronic picture investigated here can account for the $v 2$ behavior, it fails in reproducing the observed high $p_{t}$ suppression phenomena.

To illustrate to what degree rescattering modifies the high $p_{t}$ spectrum, Fig. 15 shows the ratio of cross section after rescattering divided by cross section before rescattering for three values of the impact parameter, $b=0,4$, and $8 \mathrm{fm} / \mathrm{c}$. For the most central collisions a small decrease below 1.0 is seen, but it is clear that no substantial high $p_{t}$ suppression is observed.

The partonic generator can produce jets, the thermal cannot. In Fig. 16 the jet phenomenon is demonstrated with an input of 10000 (hadronic) events from the partonic model. Particles with $p_{t}$ larger than $4.0 \mathrm{GeV} / \mathrm{c}$ are used as triggers and the distribution in azimuthal angle from the transverse direction of the trigger particle is plotted for all particles in the same event above a $p_{t}$ of $2.0 \mathrm{GeV} / \mathrm{c}$. The resulting distribution before any rescattering is shown in the figure as open circles and it can be seen that there is a small peak in the trigger direction



FIG. 16. Jet occurrence from the partonic generator. The impact parameter $b$ was $8.0 \mathrm{fm}$ and the figure is for all particle species. See the text for further explanation.

(0 deg.) and a marked sharp peak in the opposite direction, the signature of a jet (see, e.g., Fig. 29 in Ref. [19]). After rescattering (here with $\tau_{0}=0.2 \mathrm{fm}$ ) this signature still appears almost unaltered. The cases for $\tau_{0}=0.5 \mathrm{fm} / \mathrm{c}$ and $b=4 \mathrm{fm}$ and $\tau_{o}=0.2 \mathrm{fm} / \mathrm{c}$ are closely similar. The jet suppression observed experimentally is not found in the present hadronic description.

\section{SUMMARY}

Calculations of elliptic flow based on two initial state models of $\mathrm{Au}+\mathrm{Au}$ collisions at $\sqrt{s}=200 \mathrm{GeV} / \mathrm{n}$, a thermal model and a partonic model, coupled with a hadronic rescattering calculation have been presented. Results from these calculations were compared with experiments and it has been shown that both initial state models give satisfactory representations of elliptic flow measurements, provided that the rescattering is started early enough in the collision process. It is also found that the present hadronic model studies do not show the jet suppression observed experimentally.

\section{ACKNOWLEDGMENTS}

The authors in particular thank Tracy L. Smith for expert systems management at the Ohio end of the collaboration. We acknowledge financial support from the US National Science Foundation under Grant PHY-0653432 and from the Danish FNU for travel expenses.
[1] D. Teaney, J. Lauret, and E. V. Shuryak, Phys. Rev. Lett. 86, 4783 (2001).

[2] T. J. Humanic, Acta Phys. Hung. A 25, 1 (2006).
[3] H. Bøggild, O. Hansen, and T. J. Humanic, Phys. Rev. C 74, 064905 (2006).

[4] T. J. Humanic, Int. J. Mod. Phys. E 15, 197 (2006). 
[5] C. Adler et al. (STAR Collaboration), Phys. Rev. C 66, 034904 (2002); J. Adams et al. (STAR Collaboration), Phys. Rev. C 72, 014904 (2005).

[6] B. B. Back et al. (PHOBOS Collaboration), Phys. Rev. C 72, 051901(R) (2005).

[7] U. Heinz, arXiv:hep-ph/0407360 v1 (2004).

[8] D. Molnar and M. Gyulassy, Nucl. Phys. A697, 495 (2002) [Erratum-ibid. A703, 893 (2002)]; A698, 379 (2002).

[9] M. Bleicher and H. Stöcker, Phys. Lett. B526, 309 (2002).

[10] Q. Li, M. Bleicher, and H. Stöcker, Phys. Lett. B659, 525 (2008).

[11] W. Cassing and E. L. Bratkovskaya, Phys. Rev. C 78, 034919 (2008).

[12] L.-W. Chen, C. M. Ko, and Z.-W. Lin, Phys. Rev. C 69, 031901(R) (2004).
[13] Z.-W. Lin, C. M. Ko, B.-A. Li, B. Zhang, and S. Pal, Phys. Rev. C 72, 064901 (2005).

[14] L. W. Chen and C. M. Ko, Phys. Lett. B634, 205 (2006).

[15] W. H. Press, B. P. Flannery, S. A. Teukolsky, and W. T. Vetterling, Numerical Recipes in Fortran 77 (Cambridge University Press, Cambridge, UK, 1986), p. 270.

[16] W. H. Press, B. P. Flannery, S. A. Teukolsky, and W. T. Vetterling, Numerical Recipes in Fortran 77 (Cambridge University Press, Cambridge, UK, 1986), p. 273.

[17] J. D. Bjorken, Phys. Rev. D 27, 140 (1983).

[18] A. M. Poskanzer and S. A. Voloshin, Phys. Rev. C 58, 1671 (1998).

[19] J. Adams et al. (STAR Collaboration), Nucl. Phys. A757, 102 (2005).

[20] S. S. Adler et al. (PHENIX Collaboration), Phys. Rev. Lett. 91, 182301 (2003). 